\title{
Quick Response Codes to Instantiate Interactive Medical Device Instructions For Display on a Smartphone
}

\author{
M. Patterson, R. Bond, M. Mulvenna, C. Reid, F. McMahon, P. McGowan \\ Ulster University \\ Belfast, N. Ireland \\ \{m.patterson / rb.bond / md.mulvenna / ci.woodside / fc.mcmahon / p.mcgowan\}@ulster.ac.uk
}

\author{
J.McGarry, H. Cormican \\ Cirdan Imaging Ltd. \\ Lisburn, N. Ireland \\ \{jmcgarry / \\ hcormican\}@cirdan.com
}

\begin{abstract}
Usability is an increasingly important factor within the field of healthcare and medical device development. One of the main issues with the usability of medical devices is their complex nature. Therefore, it is vital that comprehensive and clear instructions are provided to aid in the operation of these devices. While paper-based instructions are commonly provided, they have many disadvantages which can be addressed by interactive digital instructions. Moreover, in an era of pervasive computing, it is important to provide these instructions at the point of need. This can be done using a Quick Response code and a smartphone which allows for interactive instructions to be instantly accessible. This paper presents a case study and a working prototype to test the utility of interactive medical device instructions accessed by a QR code attached to the medical device.
\end{abstract}

Usability, Medical Devices, Instructions, Quick Response Codes.

\section{INTRODUCTION}

Usability is an increasingly important factor within the field of healthcare and medical device development. Much research has been carried out into the usability of medical devices and how this can be improved (Zhang et al., 2003; Vincent, Li, \& Blandford, 2014; Fidler et al., 2015). Medical devices which are difficult to use and provide a poor user experience can, in extreme cases, mean the difference between life and death (Lin et al., 2001; Samore et al., 2004).

\section{THE NEED FOR INSTRUCTIONS}

One of the main issues with the usability of medical devices is their complex nature. These devices often need to facilitate multifaceted tasks, and may have a large number of steps which need to be completed. While it is important to reduce the complexity in a task process as much as possible, this can only be taken so far. Therefore, it is vital that comprehensive and clear instructions are provided to aid in the operation of these devices. Without clear instructions, user errors can occur, often with minimal fault of the user (Van Cott, 1994). This was the case in a study by Fidler et al. (2016), where a nurse failed to operate a feature without instructions. These instructions need to be provided using terminology that the user can understand, or easily learn. Analysis of a set of instructions provided with a "simple" medical device showed issues with the instructions being too complex for users to fully understand (Rogers et al., 2001). The form of which instructions take is also vitally important. Paper instructions are commonly used as they are cheap and easy to produce. They are also tangible and users can work through them at their own pace. However, there is the possibility of these instructions being misplaced or destroyed. There is also the possibility that, for particularly complex devices, having all the instructions presented on paper can be overwhelming and visually distracting for users (Figure 1). Also reading instructions and then performing instructions is a sub-optimal approach whereas hearing digital audio instructions and performing these could be more efficient for the user as evidenced in user research of the public accessible cardiac defibrillator which is a medical device that provides audio instructions in an emergency situation (Torney et al., 2016).

Providing instructions in a digital format can go a long way to combat these issues, as they are not easily destroyed or misplaced. If presented correctly, they can also better manage the cognitive load of the user, allowing the user to successfully follow the instructions. Taking this a step further, linking a Quick Response (QR) code to the digital instructions, users will be able to access 
instructions instantaneously without needing to navigate to a particular location on the web or their smart device. It also means that new users will not need any special equipment to access the instructions. Instructions themselves can be simple interactive cards augmented with audio that can be swiped to take the user through the use of a medical device in a sequential manner. Such instructions can be used in vivo for staff that have not used a particular brand of a device before or for training.

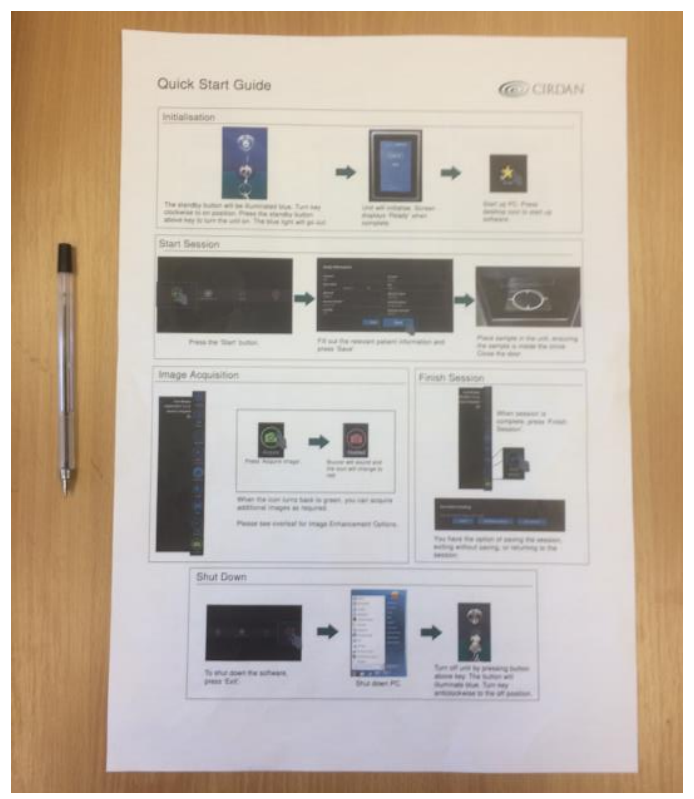

Figure 2: An example of paper instructions being overwhelming to users

\section{CASE STUDY}

A prototype of online instructions was created for a new x-ray cabinet and accompanying software. The instructions for using the device were divided into seven sections: 1) Switching on the equipment, 2) Beginning a session, 3) Loading a sample, 4)

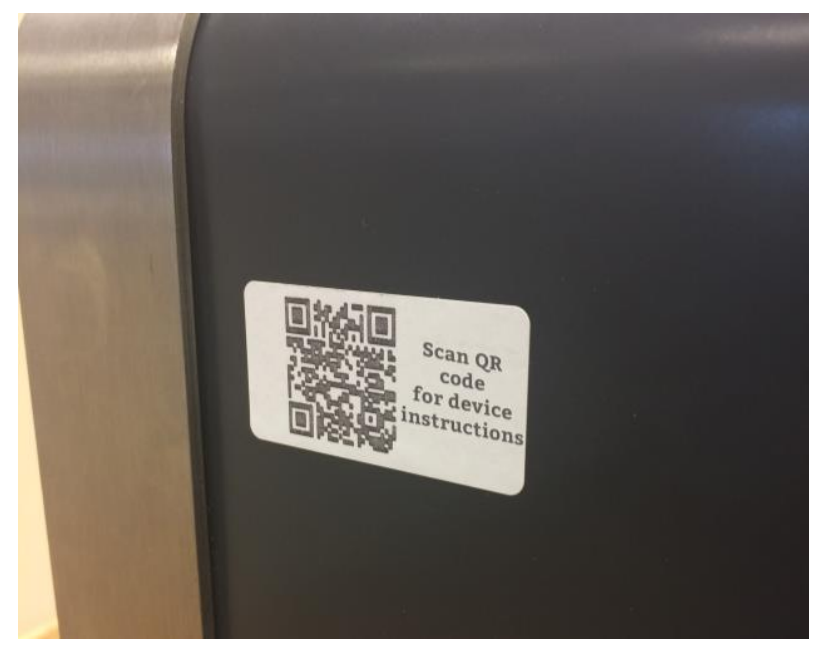

Figure 3: QR Code placed on the side of the medical device
Acquiring images, 5) Image adjustment, 6) Saving a session, 7) Switching off the equipment. Each section was broken down into a maximum of nine steps. A description, as well as an image of the $\mathrm{x}$ ray cabinet or a screenshot of the software, are used to describe what the user needs to do in order to complete the step, as shown in Figure 2.

The instructions were developed using web technologies of Hypertext Markup Language (HTML5), Cascading Style Sheets (CSS3), and JavaScript. CSS3 and JavaScript have been used to add gesture controls for ease of use on mobile devices. Transition animations were also added as these help to give the user a feeling of moving smoothly from one step to the next. CSS3 was also used to add audio instructions for each step, as hearing the instructions being read to them may be beneficial for some users. Studies have shown this is particularly true for those in the medical industry (Reid, 1987).
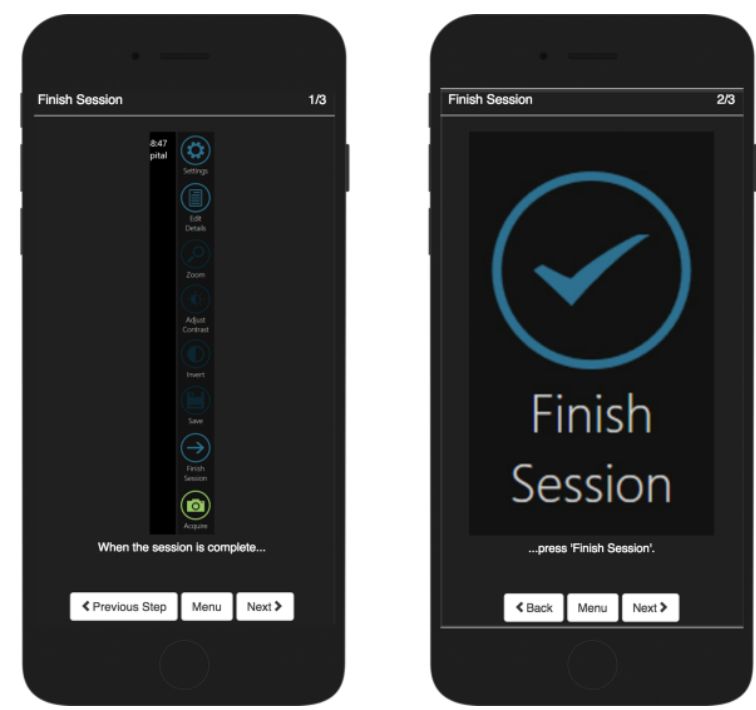

Figure 1: Examples of steps in the guide

The instructions are hosted on the company server. The QR code linking to the instructions is attached to the side of the $\mathrm{x}$-ray cabinet, as shown in Figure 3. Users can then scan the QR code with their smart phone or tablet to access the instructions.

\section{FURTHER WORK}

It is hoped that a usability study will be carried out to determine the effectiveness of the digital instructions against a paper equivalent. The medical device is to be shown at a conference where potential users can be recruited. These participants will be divided into two groups; one to use the paper version of the instructions, and the other to use the digital instructions to be accessed via the QR code. The participants will be asked to complete a series of tasks using the instructions. 
The completion times of both groups will then be analysed to determine the effectiveness of the digital instructions.

There is also potential for the digital instructions to be refactored into an augmented reality solution, as these types of instructions have proven to be even more effective than standardised digital instructions (Baird \& Barfield, 1999).

\section{REFERENCES}

Baird, K.M. and Barfield, W., 1999. Evaluating the effectiveness of augmented reality displays for a manual assembly task. Virtual Reality, 4(4), pp.250-259.

Fidler, R., Bond, R., Finlay, D., Guldenring, D., Gallagher, A., Pelter, M., Drew, B. and Hu, X., 2015. Human factors approach to evaluate the user interface of physiologic monitoring. Journal of electrocardiology, 48(6), pp.982-987.

Lin, L., Vicente, K.J. and Doyle, D.J., 2001. Patient safety, potential adverse drug events, and medical device design: a human factors engineering approach. Journal of biomedical informatics, 34(4), pp.274-284.

Reid, J.M., 1987. The learning style preferences of ESL students. TESOL quarterly, 21(1), pp.87111.

Rogers, W.A., Mykityshyn, A.L., Campbell, R.H. and Fisk, A.D., 2001. Analysis of a "simple" medical device. Ergonomics in Design, 9(1), pp.6-14.

Samore, M.H., Evans, R.S., Lassen, A., Gould, P., Lloyd, J., Gardner, R.M., Abouzelof, R., Taylor, C., Woodbury, D.A., Willy, M. and Bright, R.A., 2004. Surveillance of medical device-related hazards and adverse events in hospitalized patients. Jama, 291(3), pp.325-334.

Torney, H., O'Hare, P., Davis, L., Delafont, B., Bond, R., McReynolds, H., McLister, A., McCartney, B., Di Maio, R. and McEneaney, D., 2016. A usability study of a critical man-machine interface: Can layperson responders perform optimal compression rates when using a public access defibrillator with automated real-time feedback during cardiopulmonary resuscitation?. IEEE Transactions on HumanMachine Systems, 46(5), pp.749-754.

Van Cott, H. (1994). Human errors: Their causes and reduction. In M.S. Bogner (Ed.), Human error in medicine (pp. 53-65). Mahwah, $\mathrm{NJ}$ : Erlbaum
Vincent, C.J., Li, Y. and Blandford, A., 2014. Integration of human factors and ergonomics during medical device design and development: It's all about communication. Applied ergonomics, 45(3), pp.413-419.

Zhang, J., Johnson, T.R., Patel, V.L., Paige, D.L. and Kubose, T., 2003. Using usability heuristics to evaluate patient safety of medical devices. Journal of biomedical informatics, 36(1), pp.23-30. 